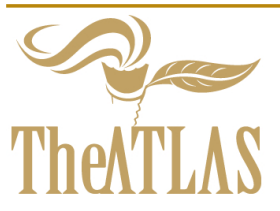

\title{
Design and Optimization of Chemical Mixing System for Vacuum Chambers: Simulation Results
}

\author{
Faruk Unker ${ }^{1}$,Erdar Kaplan ${ }^{2}$, Olkan Cuvalci ${ }^{3}{ }^{1,2}$ Department of Mechanical Engineering, Gumushane \\ University, 29100, Gumushane, Turkey, Email: farukunker@gumushane.edu.tr ${ }^{1}$, erdarkaplan@gumushane.edu.tr ${ }^{2}$, \\ ${ }^{3}$ Department of Mechanical Engineering, Karadeniz Technical University, Trabzon, Turkey Email: oculavci@ktu.edu.tr ${ }^{3}$
}

doi: $10.22545 / 2014 / 00052$

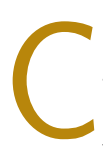
omputational fluid dynamics (CFD) is widely used in device design to determine gas flow patterns and turbulence levels. CFD is also used to simulate particles and droplets, which are subjected to various forces, turbulence and wall interactions. These studies can now be performed routinely because of the availability of commercial software containing high quality turbulence and particle models. In order to understand how the gas is brought down to wafer, it is necessary to have a knowledge of the gas flow behavior very early in the design spiral of the Tantalum nitride-Atomic layer deposition ( TaN-ALD) chamber by undertaking parametric investigation of the interaction effect between gas flow and the funnel structure. This paper presents such a parametric investigation on a generic TaN-ALD chamber using CFD. The results presented have been analyzed for a total of 11 different cases by varying neck and nozzle angles for a process gas. The gas flow was mainly investigated for the nozzle angles of $4.5^{\circ}, 9^{\circ}, 12^{\circ}$ and $20^{\circ}$ and the film thickness results were compared with numerical flow patterns. CFD simulations using the turbulence model in ANSYS Fluent v.13 are undertaken. The parametric study has demonstrated that CFD is a powerful tool to study the problem of gas flow-structure interaction on funnel and is capable of providing a means of visualizing the path of the gas under different operating conditions.

Keywords: Chemical Mixing System, Vacuum Chambers, flow-structure interaction, funnel design.

\section{Introduction}

Computer-based simulation is now widely used across all branches of science and engineering and is finding ever more applications as computing power becomes cheaper and commercially available software becomes more powerful. Here we review some of the many studies of the design of TaN-ALD Chamber funnel that have used computational modeling of gas flow and particle dynamics to optimize gas delivery. It is worth noting that computational tools are used widely in the manufacture of the actual device, where Finite Element Analysis (FEA) is used to ensure that the device has sufficient strength without being over-engineered and wasting valuable material. 
The prediction of flow path of gas through the chamber funnel is extremely complicated since the phenomenon is affected by a large number of parameters like gas velocity and direction, level of turbulence, geometry of the structures on the funnel, efflux velocity of gas etc. [1-3]. To complicate the matters, the entire turbulent flow field is subject to abrupt changes as the nozzle angle alters. It is not always possible to cover the entire range of all parameters to simulate every possible working condition in the funnel $[4,5]$. CFD has emerged as a serious alternative to funnel studies and is capable of providing solutions very early in the design. This paper presents the results of parametric investigation of the interaction effect between the gas flow and the nozzle angle of the funnel (Figure 1) using CFD by varying the parameters of lid shape [6-9]. Four variants of the nozzle angles (Figure 2) were investigated at four funnel types each and further, each of these combinations were investigated at two different onset flow conditions. The results are from the numerical studies of typical cases (Case 1, 9, 10 , and 11) undertaken with an aim of gaining an understanding of the typical flow field around the topside of wafer and the interaction between the shape (combination of nozzle and neck angles of the funnel, making a total of 11 cases) and the funnel's gas flow.

Realizing of non-uniform film thickness of wafer is therefore an important aspect of funnel design that falls under the category of TaN-ALD Chamber. However, very often, this application of TaN-ALD Chamber is not recognized a priori in the design of funnel. In order to take the film thickness problem into account, the vacuum chamber designer needs to be able to have a means of visualizing the path of the funnel under different conditions during the design phase, which will enable detection of shortfalls very early in the design spiral. This requires the vacuum chamber designer to have knowledge of gas flow behavior through the TaN-ALD Chamber, which shall enable him to find efficient means to eliminate the problem and also to avoid the costly post construction additions and alterations. Traditionally, the funnel performance has been investigated using ANSYS fluent in a vacuum chamber at a relatively advanced stage of design, when many aspects of the design are frozen.

\section{Simulations Conditions}

CFD simulations were conducted to determine the flow of the gas through the funnel lid and the subsequent film thickness on wafer. In general, flow fields were simulated using commercial CFD codes, with the majority of researchers utilizing ANSYS CFX or ANSYS Fluent. CFD simulations also identify the uniformity of the film thickness of the wafer when the flow pattern of the process gas was changed. However increasing the rotating of flow for any given process gas could provide this uniformity well. Furthermore, by altering design parameters, such as nozzle angle and neck diameter will allow the simulation of gas at a size range suitable for funnel delivery, ensuring mixture within the funnel airways.

In general, flow fields were simulated using commercial CFD codes of ANSYS CFX or ANSYS Fluent.The k- model or the Shear Stress Transport (SST) model was usually used for turbulence modelling. It is substantial that most researchers have used the k- or SST models which have the capability to be applied throughout the turbulent boundary layer. Launder and Spalding (1974) [10] and Menter (1994) [11] provide more details on these turbulence models. The specific CFD code and turbulence modelling utilised by each researcher is outlined in Table 1 .

\section{Results and Discussions}

In order to identify the underlying effects of funnel shape on the turbulence intensities generated in the vacuum chamber, it is necessary to analyze the flow field results obtained from CFD measurements . Since nozzle angle is found to have important effect on the turbulence intensity inside the vacuum chamber, the flow field results of the different shapes of nozzle are used for more detailed analysis. Figure 1 shows the flow field results obtained from front cross-section measurements of nozzle. The color contours in the figures illustrate the magnitude of the turbulence intensity from low (blue)to high (red) levels. The direction and size of the vectors represent the direction and magnitude of the gas flow at each particular node.

CFD was utilized to simulate the mixture of the gas spray in order to determine the best operating conditions. Specifically, we investigated the influence of velocity vectors and gas paths, as well as the role of the nozzle angle on the mixture of gas 
Table 1: Summary of CFD solvers turbulence model used.

\begin{tabular}{lll}
\hline CFD code/software & Turbulence model & Reference number \\
\hline ANSYS CFX & $\mathrm{k}-\varepsilon$ & {$[12]$} \\
& $\mathrm{SST}$ & {$[1321]$} \\
& $\mathrm{k}-\omega$ & {$[22]$} \\
ANSYS Fluent & $\mathrm{k}-\omega$ & {$[12,2225]$} \\
& $\mathrm{k}-\varepsilon$ & {$[12]$} \\
& Laminar flow & {$[2628]$} \\
& Not specified but used & {$[29,30]$} \\
CFD-ACE + & Laminar flow & {$[31,32]$} \\
\hline
\end{tabular}

Table 2: Optimization of dimensions.

\begin{tabular}{ccccccccc}
\hline Case & $\begin{array}{c}\text { Nozzle } \\
\text { Angle, } \alpha \\
{[\mathrm{deg}]}\end{array}$ & $\begin{array}{c}\text { Neck } \\
\text { angle } \\
{[\mathrm{deg}]}\end{array}$ & $\Phi[$ inch $]$ & $\begin{array}{c}\text { Lid fillet } \\
\mathrm{R}[\text { inch }]\end{array}$ & $\begin{array}{c}\mathrm{H} 1 \\
{[\text { inch }]}\end{array}$ & $\begin{array}{c}\text { Flow } \\
\text { pattern }\end{array}$ & $\begin{array}{c}\text { Center non- } \\
\text { uniformity }\end{array}$ & $\begin{array}{c}\text { Overall non- } \\
\text { uniformity }\end{array}$ \\
\hline 1 & 12 & 4.5 & 0.55 & 0.0 & 2.0 & Rotating & 3.7 & 9.9 \\
2 & 8 & 4.5 & 0.55 & 0.6 & 2.0 & Impinging & 5.5 & 17.5 \\
3 & 8 & 4.5 & 0.55 & 0.25 & 2.0 & Impinging & 6 & 18 \\
4 & 8 & 4.5 & 0.65 & 0.25 & 2.0 & Rotating & 5.4 & 12.7 \\
5 & 14 & 4.5 & 0.55 & 0.6 & 2.0 & Rotating & 3.2 & 10.2 \\
6 & 20 & 4.5 & 0.55 & 0.6 & 2.0 & Rotating & 3.6 & 9.7 \\
7 & 30 & 4.5 & 0.55 & 0.6 & 2.0 & Rotating & 4.4 & 10.1 \\
8 & 4.5 & 4.5 & 0.57 & 0.6 & 1.5 & Rotating & 3.6 & 9.8 \\
9 & 12 & 9 & 0.55 & 0.7 & 2.0 & Rotating & 1.4 & 8.0 \\
10 & 20 & 12 & 0.55 & 1.2 & 2.0 & Rotating & 2.5 & 7.4 \\
11 & 12 & 20 & 0.55 & 0.7 & 2.0 & Rotating & 2.6 & 7.9 \\
\hline
\end{tabular}

flow. Table 2 shows the presence of 11 typical cases, respectively that involves the flow pattern, center non-uniformity and overall uniformity. It was found that changing the nozzle angle through the funnel altered the level of uniformity, optimal performance was achieved at rotating flow pattern, but the improvement is limited with wafer when the funnel type was changed further. In Figure 3, the rotating flow pattern of the gas was found to run in with the precursor consumption flux distributions agreeing with measured film thickness map, and increasing neck angle was found to decrease the uniformity of center but the overall non-uniformity of the film thickness remained unchanged.

The shape of funnel causes funnel gases to disperse downward toward the center more rapidly than side. This has many adverse consequences like the nonuniformity of film thickness. The existing fillet of lid played no significant effect on the performance or the flow field generated and widening the fillet of lid (R) made little difference to the uniformity perfor- mance, but reduced the angle of the nozzle leading to changed flow pattern. Flow patterns with a wide nozzle angle were found to be more easily rotating; however the effect of lid fillet size was less significant than the nozzle angle size, especially at high flow velocities [33, 34]. Interestingly, nozzle angle of $12^{\circ}$ with neck angle of $20^{\circ}$ was found to be important to film uniformity. On the other hand, uniformity efficiency was found to be directly proportional to the ratio of the flow pattern $[8,34]$.

\subsection{Effect of Funnel Lid Dimensions on Gas Velocity Vectors [m/s]}

Figure 1 shows the variation of gas velocities with the nozzle angle of the funnel. The present simulation is carried out with four nozzle angles. The lid has been shaped with the angles of $4.5^{\circ}, 9^{\circ}, 12^{\circ}$ and $20^{\circ}$, respectively. It can be seen from the Figure 1 that the reverse flow initially decreases after the angle of $9^{\circ}$ and then is mostly eliminated with the angle 


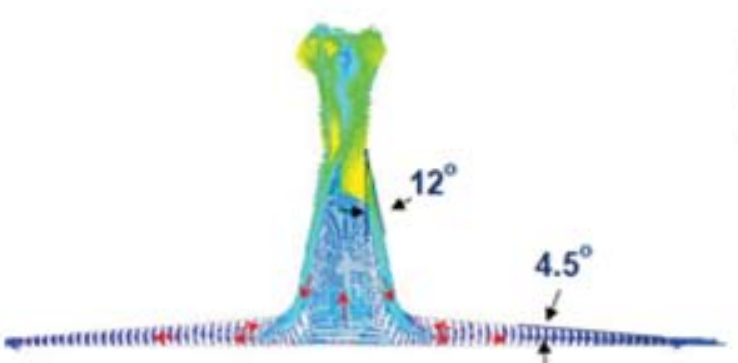

(a)

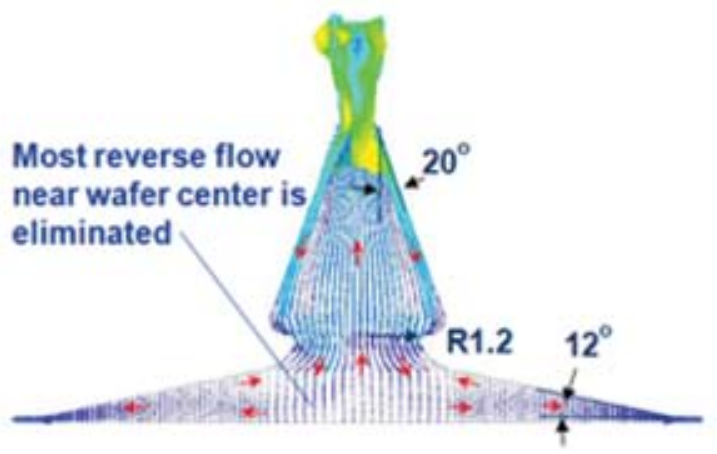

(c)

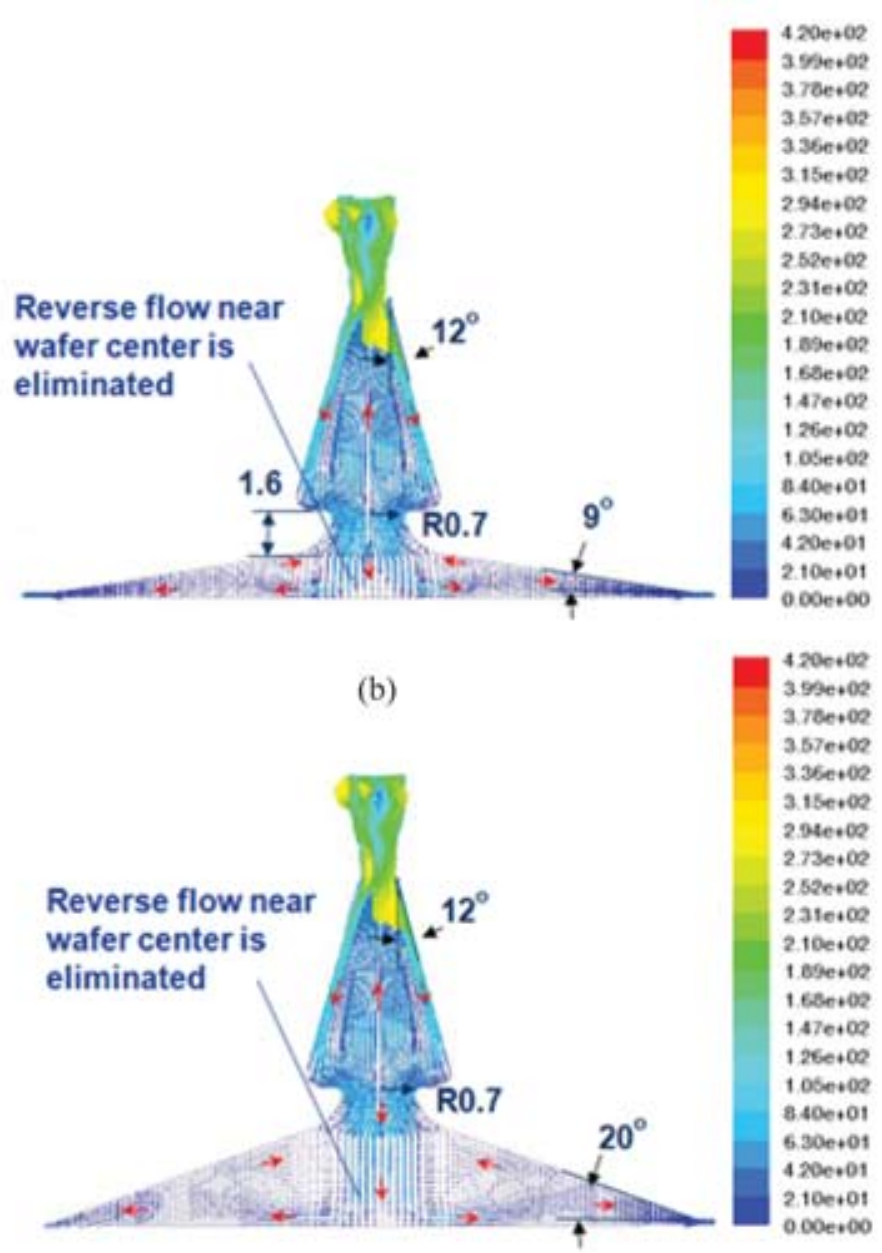

(d)

Figure 1: Gas Velocity Vectors [m/s] (a) Case 1, Current Hardware, (b) Case 9, R0.7" Neck, 9, (c) Case 10, R1.2" Neck, $12^{\circ}$, (d) Case 11, R0.7” Neck, $20^{\circ}$.

of $12^{\circ}$, suggesting the existence of an optimum lid angle. The center line velocity drop is significant when the nozzle angle is $12^{\circ}$, so there is less nonuniform film thickness near the center of wafer for this case [18]. But after the nozzle angle of $12^{\circ}$ the center line velocity vector becomes significant and the gas velocity near the wafer center rises. Even though some reverse flow is observed, the absence of large variation velocity fields gives rise to only small turbulence intensity. Both the increases in gas velocity and decreases in reverse flow are stronger on the wafer with good uniformity because of the decreased reverse flow streams from the turbulence [16].

Similar change in flow patterns is also observed using other nozzle angles as shown in Table 2. A comparison of the flow fields among Fig 2(a)(b) also confirms that an increase in angle from $4.5^{\circ}$ to $12^{\circ}$ amplifies the formation of turbulence intensities. The distinctive difference between the rotating and impinging flow patterns thus give rise to a high turbulence intensity shows that as the process gas travels from the inlet, the expansion motion of gas becomes more irregular $[16,18,35]$. Reverse gas flow can also be observed near wafer center. Particularly, path lines near the center of the wafer are mixed with the vortex. The vortex generated is also spreading from initially the central region to the entire wafer. Additional vortices are generated from the converging flow pattern and hence the turbulence intensity is further improved. As a result, the turbulence intensity increases because of the changed shape parameters of funnel lid. 


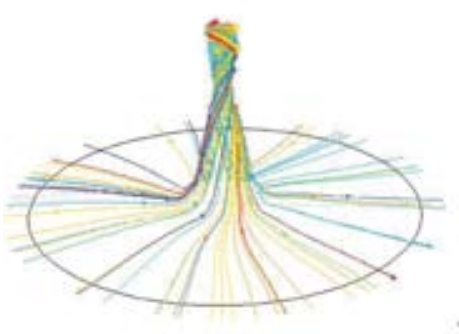

(a)

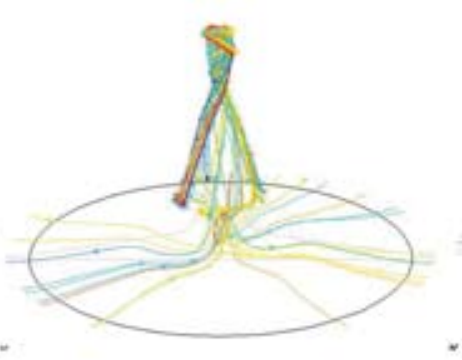

(b)

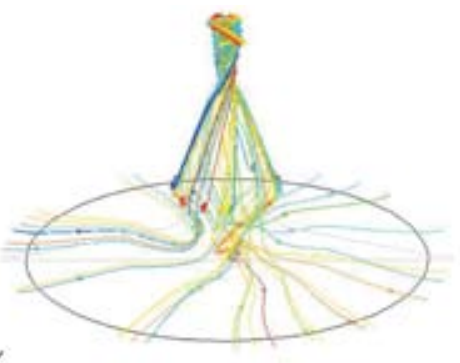

(c)

Figure 2: Effect of funnel lid dimensions on Gas Path Lines, (a) Case 1, Current Hardwareb) Case 9, R0.7" Neck, $9^{\circ}$, (c) Case 10, R1.2" Neck, $12^{\circ}$.

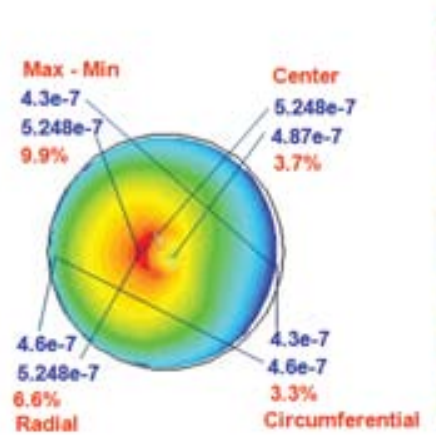

(a)

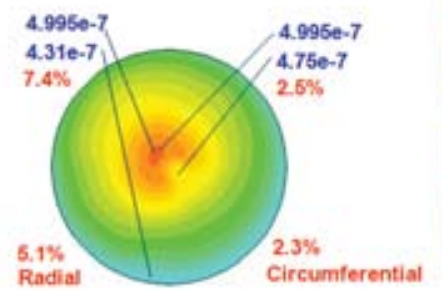

(c)
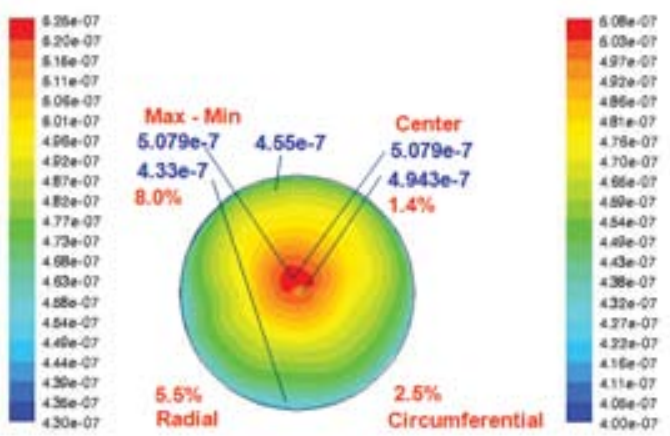

(b)
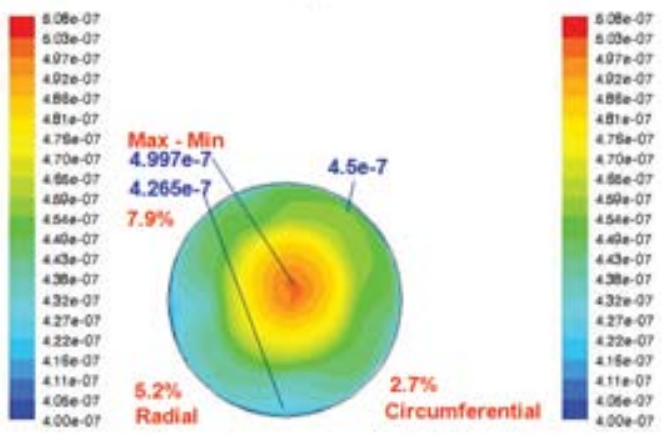

(d)

Figure 3: Consumption Flux $\left[\mathrm{kg} /\left(\mathrm{m}^{2} \mathrm{~s}\right)\right]$ of Process Gas, (a) Case 1, Current Hardware, (b)Case 9, R0.7" Neck, 9, (c) Case 10, R1.2" Neck, 12 ${ }^{\circ}$, (d) Case 11, R0.7" Neck, $20^{\circ}$.

\subsection{Effect of Funnel Lid Dimensions on Consumption Flux $\left[\mathrm{kg} /\left(\mathrm{m}^{2} \mathbf{s}\right)\right]$ of Process Gas}

The simulation is carried out for four nozzle angles located at the funnel lid as shown in Figure 3. The analysis is also carried out for four cases as has been shown in Figure 3 which demonstrates the effect of nozzle angles on consumption flux of the process gas. The nozzle angles considered for the CFD analysis were $4.5^{\circ}, 9^{\circ}, 12^{\circ}$, and $20^{\circ}$. In the 1 st case the consumption flux on the wafer is kept $9.9 \%$ from $\max$ to $\mathrm{min}$, in 2 nd case the flux of the center is $8.0 \%$ to the circumferential and in 3rd case the consumption flux on the wafer is kept $7.4 \%$ from max to min. When the nozzle angle is $9^{\circ}$ the center flux becomes the best uniformity but the overall uniformity on the wafer is still lower compared to the case of a nozzle angle of $12^{\circ}$ because the consumption flux along the centerline of the wafer is too quick for the 


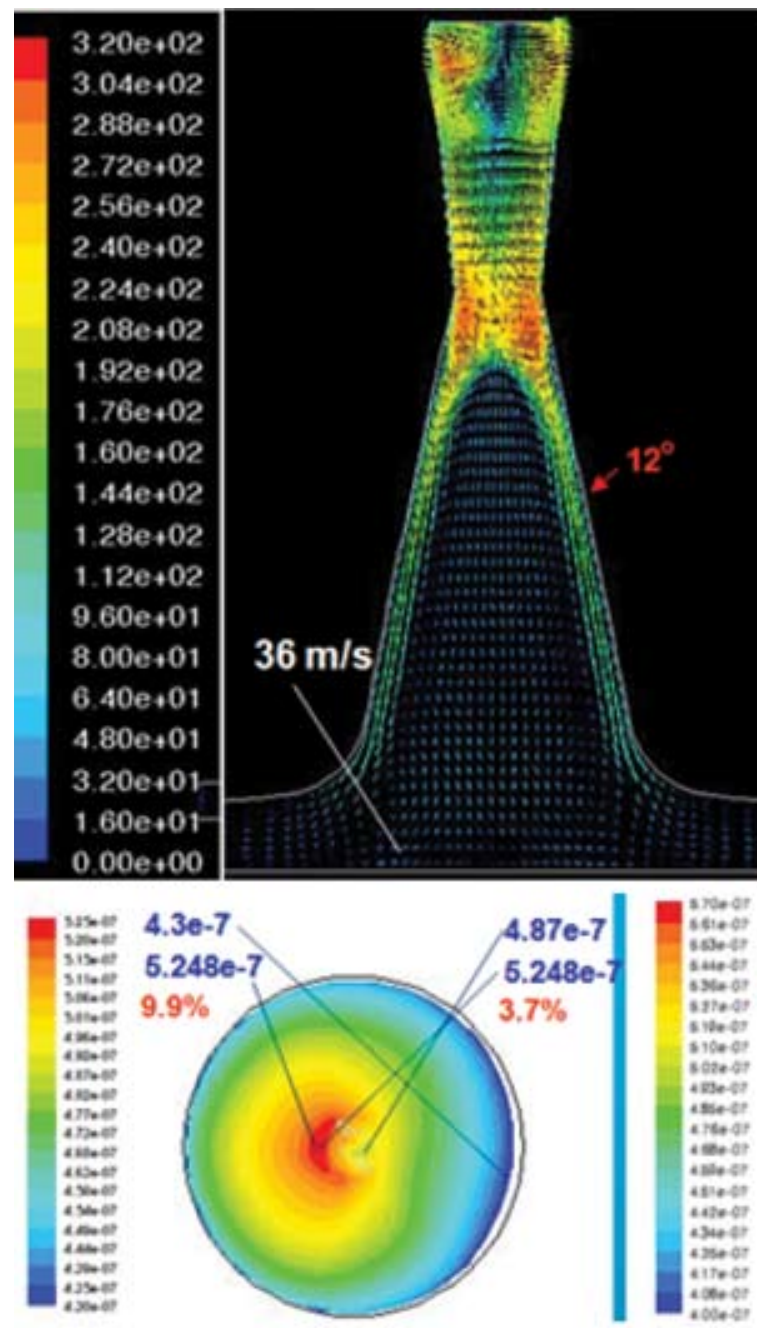

(a)
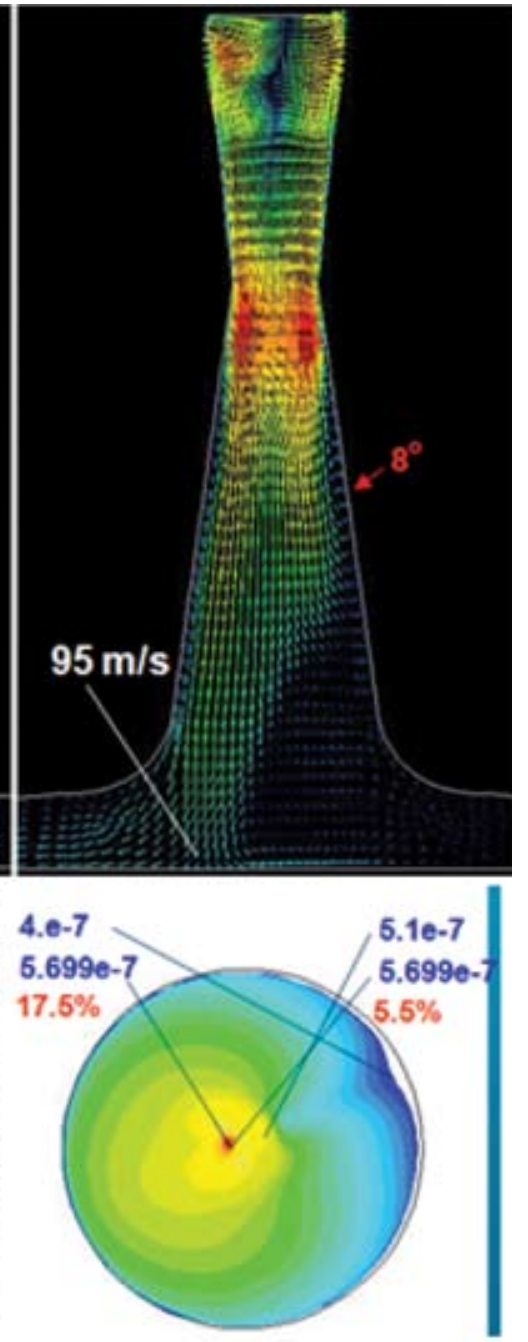

(b)

Figure 4: Comparison between Simulated Precursors: Velocity Vector $[\mathrm{m} / \mathrm{s}]$ and Consumption Flux of Process Gas a)Case 1, Rotating Flow (Current Hardware) b)Case 2, Impinging Flow.

case of nozzle angle of $9^{\circ}$. Since the funnel becomes narrow, the viscous effect becomes prominent and the pressure rises quickly along the axis of the funnel causing less gas ingress into the funnel. So, there is the existence of a highest turbulence for optimum consumption flux into the funnel. Optimal performance was achieved at a working shape of Case 10 which generated center non-uniformity with a mean percentage of 3.7 at an overall non-uniformity of 9.9 percentage. This can be explained from the fact that the reverse flow velocity near the center of wafer is not increased significantly as rotating flow velocity through the funnel is increased. However, neck angle on the funnel is found to have negligible effect on the turbulence intensity generated [6]. It is also concluded from Figure 3 that the variation of neck angle does not contribute significantly to the change of radial uniformity.

The variation of flow pattern due to the inclination of the nozzle angle is shown in Table 3 and rotating flow pattern is preferred as impinging flow shown in Figure 4 cause non-uniformity. As a matter of fact, a comparison of the velocity vector in the side cross-sections shown in Figure 4 indicates that the co-presence of high velocity streams and low velocity of reverse flow diminishes the effect of non-uniformity of film thickness as the turbulence intensity is a measure of the difference in the velocity fields. At narrow nozzle angle condition, a certain portion of turbulence intensity is contributed by the change in gas velocity due to the substantial change in flow area. However, at wide nozzle angle 
conditions in Figure 4, the reduction in flow velocity becomes less significant that the turbulence intensity is essentially governed by the reverse flow streams. An interpretation of both vertical and horizontal cross-sectional views together suggests a spiral flow generated inside the vacuum chamber. This is because a large velocity gradient is generated due to the sudden expansion in the flow area as the gas flows pass the nozzle. As a result, turbulence is generated and intensifies along the axial location before reaching a fully developed flow [6], [18].

\subsection{Effect of Funnel Lid Dimensions on Mole Fraction of Process Gas on Wafer Variation with Time}

Figure 5 shows the comparison of the mole fractions of two cases. It can be seen that the purge duration increases to $245 \mathrm{~m} / \mathrm{s}$ from $98 \mathrm{~m} / \mathrm{s}$. It is evident from Fig. 6 that the purging is influenced with the inclination of the nozzle angle. It can be seen from the plot the mole fraction of the process gas decreases into the funnel as the nozzle angle increases. High nozzle angle creates a low pressure field around the funnel [35]. Due to this low pressure field, the difference in pressure across the funnel (inside and outside the funnel) decreases causing less suction of gas from the funnel. Therefore, it can be concluded the entrainment of mole fraction of the process gas in the funnel decreases with increase in angle on nozzle of the funnel.

\section{Conclusion}

The results of this work show that the prominent application of modern simulation methods such as process simulation and CFD is an effective tool for the optimization and design of an innovative concept for the gas flow of funnels. Generally, these tools enable the simulation and analysis of numerous complex processes in the chemical engineering practice in an early stage of the project. The application of powerful calculation tools with a sound physical basis combined with the reduction of experimental efforts result in lower development costs and a shorter time-to-market of new processes and apparatuses. The results of the flow calculations as well as the results generated with the CFD simulations mostly show very good agreement with film uniformity of the wafer, which can be used for the refinement of models and modeling the funnel parameters of a ALD chamber.

The work reported in this paper is a complete numerical investigation of parametric study of gas flow through the funnel of a ALD chamber. Throughout the entire CFD analysis vacuum conditions has been considered to be constant in the chamber. The following conclusions are drawn from the results of the present study:

- The performance of the funnel flow in the chamber is found to recover at the nozzle angles between $9^{\circ}$ and $12^{\circ}$ and therefore, these may be termed as "favorable range of nozzle angles" as far as uniform film at the wafer is concerned. It is strongly recommended that the funnel designer should specially study the flow conditions with relative flow patterns over the favorable range of nozzle angles.

- The reverse flow initially decreases after the angle of $9^{\circ}$ and then is mostly eliminated with the angle of $12^{\circ}$, suggesting the existence of an optimum funnel nozzle angle. This can be explained from the fact that the reverse flow velocity near the center of wafer is not increased significantly as rotating flow velocity through the funnel is increased.

- Rotating flow pattern is preferred as impinging flow cause non-uniformity. The rotating flow increases with increase in nozzle angle. Center thin map is caused by imperfect mixing and flow pattern with funnel.

- The uniformity rate is independent of amount of fillet radius but strongly depends on nozzle angle of funnel. The study clearly indicates that gas flow is not influenced with the fillet radius of the funnel but depends a bit on the neck angle of the funnel. Therefore the cooperation of the gas flow pattern with the neck angle is not negligible and the neck angle should always be considered in such calculations.

- The purging time of the process gas increases into the funnel as the nozzle angle rises. From the CFD analysis it has become clear that increasing the nozzle angle of the funnel, the purging time is increased. But increase in purging time of the chamber is limited due to design of funnel as well as from economical point of view. Film uniformity may be improved by optimizing 
Table 3: Breakdown of depth non-uniformity.

\begin{tabular}{lcl}
\hline Non-uniformity break down & Non-uniformity [\%] & Improvement method \\
\hline Center low & 3.7 & Eliminate reverse flow \\
Radial gradient & 6.6 & Introduce precursor to edge \\
Circumferential gradient & 3.3 & Enhance mixing \\
\hline
\end{tabular}

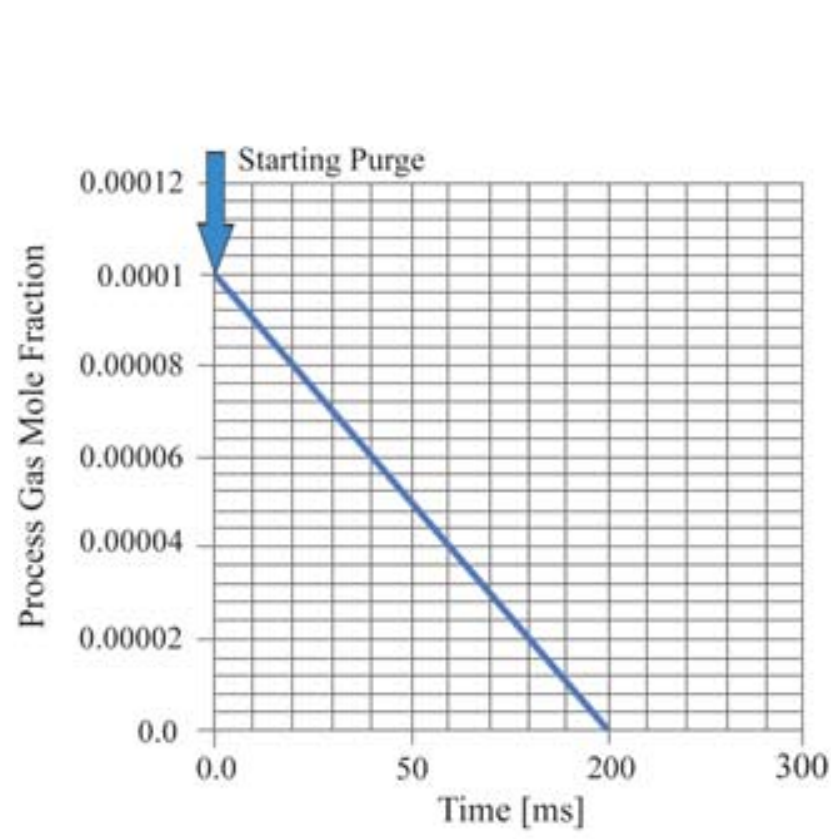

(a)

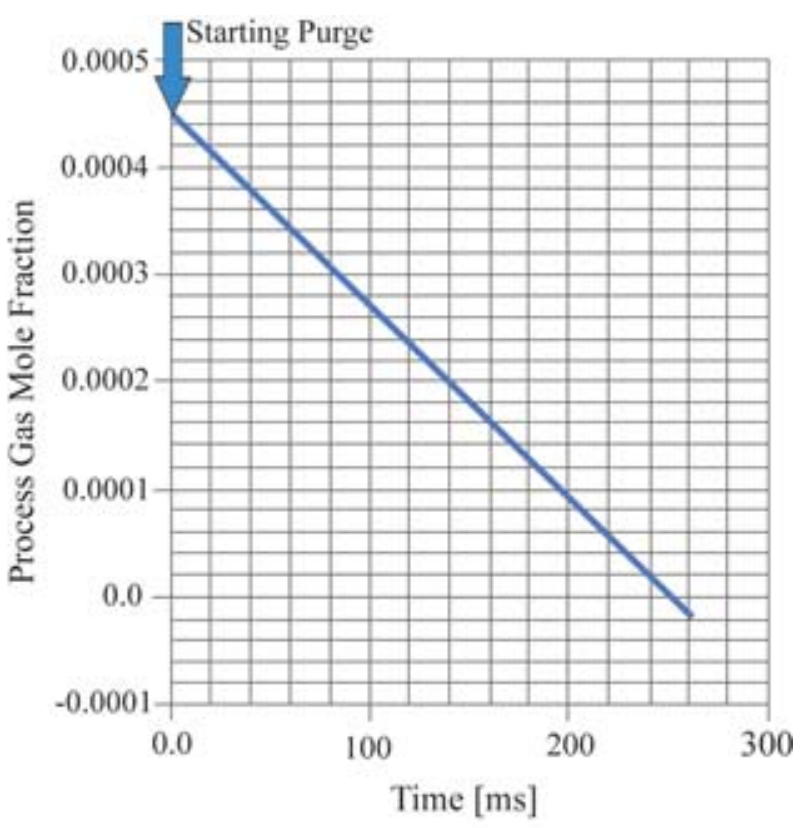

(b)

Figure 5: (a) Case 1, Current Hardware (Case 1: $98 \mathrm{~ms}$ to reduce the process gas concentration by 5 orders) (b) Case 10, R1.2" Neck, $12^{\circ}$ (Case 10: $245 \mathrm{~ms}$ to reduce the concentration by 5 orders at wafer edge (center)).

the dimensions, but the improvement is limited with the purging time cost. But it is suggested that the maximum possible funnel nozzle angle of $12^{\circ}$ should be used.

- Simulated precursor consumption flux distributions agree with measured film thickness map.

- Strong correlation between simulated gas flow patterns (re-circulating flow, vortex) and onwafer deposition pattern. Re-circulating of the flow has improved the wafer deposition efficiency. It seems that this is mainly due to the vortex of gas flow, due to increasing the re-circulating flow in which the vortex takes place.

- With concise mathematical models at hand, process simulation and CFD provide efficient and cost-saving assistance for chemical engineers and vacuum chamber constructors. CFD methods allow complex flow patterns around the cham- ber to be identified and problems about gas flow can now be addressed during the early stages of chamber design. CFD also provides capabilities for problem troubleshooting and solving of the gas flow problem on the already constructed and operational vacuum chambers.

- This study has demonstrated that CFD is a powerful tool capable of predicting the features of the gas flow-structure interaction. As a flow simulation tool, CFD can predict gas flow structure interaction throughout the funnel, which is particularly advantageous for the investigation of ALD Chambers with complex structures. Further, the results of the study show that level of detail and the realistic physical foundations of the CFD simulation of the flow pattern and dispersal of gas flow over the wafer gives unique opportunities to chamber designers that were not available before. 


\section{References}

[1] ISO/DIS 3570/1, 1975.

[2] Poulter K.F., 1977. The calibration of vacuum gauges. J. Phys. E: Sci. Instrum., vol. 10, pp.112.

[3] Szwemin P., 2004. The influence of the blocking plate diameter on the gas flux distribution in the calibration chamber. Vacuum, vol. 73, pp.263.

[4] Maev R., and Leshchynsky V., 2008. Introduction to low pressure gas dynamic spray. Weinheim, Germany: Wiley-Vch.

[5] Cai C., and Boyd I.D., 2007. Collisionless gas flow expanding into vacuum. Journal of Spacecraft and Rockets, vol.44, pp.1326-1330.

[6] Versteeg H.K., and Malalasekera W., 1995. An introduction to computational fluid dynamics. The Finite Volume Approach, Addison Wesley Longman, Harlow, UK.

[7] Tu J., Yeoh G.H., and Liu C., 2008. Computational fluid dynamics. A practical approach, Elsevier, Oxford, UK.

[8] Patankar S.V., 1980. Numerical heat transfer and fluid flow. Series in Computational Methods in Mechanics and Thermal Sciences, Taylor and Francis.

[9] Ferziger J.H., and Peric M., 2002. Computational Methods for Fluid Dynamics.

[10] Launder B.E., and Spalding D.B., 1974. The numerical computation of turbulent flows. Comput. Meth. Appl. Mech. Eng. vol.3, pp. 269289.

[11] Menter F.R., August 1994. Two-equation eddyviscosity turbulence models for engineering applications. AIAA J., vol32, pp.15981605.

[12] Versteeg H.K., Hargrave G., Harrington L., Shrubb I., and Hodson D., 2000. The use of computational fluid dynamics (CFD) to predict pMDI air flows and aerosol plume formation. Respiratory Drug Delivery VII, vol.1, pp. 257264.

[13] Lai Jr. M., Huang C.Y., Chen C.H., Linliu K., and Lin J.D., 2010. Influence of liquid hydrophobicity and nozzle passage curvature onmicrofluidic dynamics in a drop ejection process. J. Micromech. Microeng. vol. 20, 015033, p.14.

[14] Nichols S.C., and Wynn E., 2008. New approaches to optimizing dispersion in dry powder inhalers - dispersion force mapping and adhesion measurements. Respir. Drug Deliv., vol. 1, pp. 175184.
[15] Coates M.S., Chan H.-K., Fletcher D.F., and Raper J.A., 2005. Influence of air flow on the performance of a dry powder inhaler using computational and experimental analyses. Pharm. Res., vol. 22, pp. 14451453.

[16] Coates M.S., Fletcher D.F., Chan H.-K., and Raper J.A., 2004. Effect of design on the performance of a dry powder inhaler using computational fluid dynamics. Part 1: grid structure and mouthpiece length, J. Pharm. Sci., vol. 93, pp. 28632876.

[17] Coates M., Chan H.-K., Fletcher D. F., and Chiou H., 2007. Influence of mouthpiece geometry on the aerosol delivery performance of a dry powder inhaler. Pharm. Res., vol. 24, pp. 14501456.

[18] Coates M.S., Chan H.-K., Fletcher D.F., and Raper J.A., 2006. Effect of design on the performance of a dry powder inhaler using computational fluid dynamics. Part 2: air inlet size. J. Pharm. Sci., vol. 95, 13821392.

[19] Calvert G., Ghadiri M., and Tweedie R., 2009. Aerodynamic dispersion of cohesive powders: a review of understanding and technology. Adv. Powder Technol.,vol. 20, pp. 416.

[20] Wong W., Fletcher D.F., Traini D., Chan H.K., Crapper J., and Young P.M., 2010. Particle aerosolisation and break-up in dry powder inhalers 1: evaluation and modelling of venturi effects for agglomerated systems. Pharm. Res., vol. 27, pp. 13671376.

[21] Wong W., Fletcher D.F., Traini D., Chan H.-K., Crapper J., and Young P.M., 2011. Particle aerosolisation and break-up in dry powder inhalers: evaluation and modelling of impaction effects for agglomerated systems. J. Pharm. Sci.,vol. 100, pp. 27442754.

[22] Kleinstreuer C., Shi H., and Zhang Z., 2007. Computational analyses of a pressurized metered dose inhaler and a new drugaerosol targeting methodology. J. Aerosol Med., vol.20, pp. 294309.

[23] Longest P.W, Hindle M., Das Choudhuri S., and Xi J., 2008. Comparison of ambient and spray aerosol deposition in a standard induction port and more realistic mouththroat geometry. J. Aerosol Sci., vol. 39, pp. 572591.

[24] Longest P.W, Hindle M., Choudhuri S. D., and Byron P.R., 2007. Numerical simulations of capillary aerosol generation: CFD model development and comparisons with experimental data. Aerosol Sci. Technol., vol 41, pp. 952973.

[25] Longest P.W, Hindle M., Choudhuri S. D., 2009. Effects of generation time on spray aerosol transport and deposition in models of the mouththroat 
geometry. J. Aerosol Med. Pulm., vol. D 22, pp. 6784 .

[26] Longest P.W, Hindle M., 2009. Quantitative analysis and design of a spray aerosol inhaler. Part 1: effects of dilution air inlets and flow paths. J. Aerosol Med. Pulm., vol 22 (3), pp. 271-283.

[27] Amirav I., and Newhouse M.T., 2001. Aerosol therapy with valved holding chambers in young children: importance of the facemask seal. Pediatrics, vol. 108, pp. 389394.

[28] Shen S.-C., Wang Y.-J., and Chen Y.-Y., 2008. Design and fabrication of medical micronebulizer. Sens. Actuators, vol. 144, pp. 135143.

[29] Coates M.S., Fletcher D.F., Chan H.-K., and Raper J.A., 2004. A comparative study of two marketed pulmonary drug delivery devices using computational fluid dynamics. Respiratory Drug Delivery IX, vol. 3, pp. 821824 .

[30] Tibbatts J., Mendes P.J., and Vlllax P., 2010. Understanding the power requirements for efficient dispersion in powder inhalers: comparing CFD predictions and experimental measurements. Respir. Drug Deliv., vol. 1, pp. 323330.

[31] Arulmuthu E.R., Williams D.J., Baldascini H., Versteeg H.K., and Hoare M., 2007. Studies on aerosol delivery of plasmid DNA using a mesh nebulizer. Biotechnol. Bioeng., vol. 98, pp. 939955.

[32] Jeng, Y.R. Su C.C., Feng G.H., and Peng Y.Y., 2007. An investigation into a piezoelectrically actuated nebulizer with $\mu$ EDM-made micronozzle array. Exp. Therm Fluid Sci., vol. 31, pp.11471156.

[33] Zhu H., Zhou Z., Yang R., and Yu A., 2007. Discrete particle simulation of particulate systems: theoretical developments. Chem. Eng. Sci., vol. 62, pp. 33783396 .

[34] Zhu H.P., Zhou Z.Y., Yang R.Y., and Yu A.B., 2008. Discrete particle simulation of particulate systems: a review of major applications and findings. Chem. Eng. Sci., vol. 63, pp. 57285770.

[35] Coates M., Chan H.-K., Fletcher D., and Chiou H., 2007. Influence of mouthpiece geometry on the aerosol delivery performance of a dry powder inhaler. Pharm. Res., vol. 24, pp. 14501456.

\section{About the Authors}

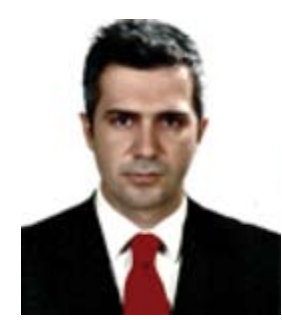

Faruk Unker is currently a Research Assistant in Mechanical Engineering at Gumushane University. He received his B.Sc. and M.Sc. in Mechanical Engineering from Karadeniz Technical University (KTU) in 2007 and 2012, respectively. Faruk Unker presently continues his Ph.D. education at KTU. As a Ph.D. student his research efforts have focused on Manufacturing, Quality Improvement, and Environmental Issues Related to Design and Manufacturing. His industrial experience includes product design and development, manufacturing supervision, quality assurance and facility project coordination. He worked for an air-spring manufacturing company in Turkey as a Technical Service Engineer.

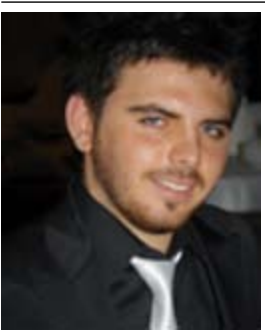

Erdar Kaplan Erdar Kaplan is currently a Lecturer in Mechanical Engineering at Gumushane University. He received B.Sc. degree in Department of Machine at Technical Education Faculty from Afyon Kocatepe University (AKU), Afyon, Turkey in 2009 and M.Sc. degree in Mechanical Engineering from AKU in 2012. He presently continues his Ph.D. education at Karadeniz Technical University (KTU). His research interests include design and manufacturing, development of materials and alloy design.

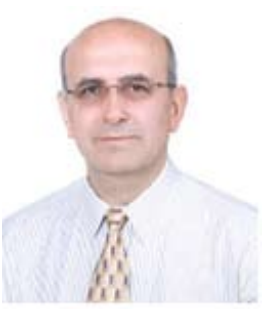

Dr. Olkan Cuvalci is is Professor at the Technical University of Karadeniz, in the Department of Mechanical Engineering. He received his B.Sc. and M.Sc. in Mechanical Engineering at the Karadeniz Technical University (KTU) in 1983 and 1985, respectively, and Ph.D. in Mechanical Engineering from Texas Tech University in 1992. From 1986-1993 he worked at KTU as a Research Assis- 
tant at the faculty of engineering. From 1996-1999 Cuvalc worked as a research associate in Mechanical Engineering at Texas Tech University. From 1999-2000 he worked at the Western Kentucky University as an Associate Professor at the Engineering of Technology. In 1993, he became an Assistant Professor at KTU, in the Department of Mechanical Engineering. He was promoted to Associate Professor in 1995 and to Full Professor in 2012. From 2009-present he is also an Adjunct Professor at Texas Tech University. Professor Cuvalc is presently the Dean of Karadeniz Technical University's Faculty of Engineering. His several research interests lie at the intersections of control and optimization theory, Test Method Development, Experimental \& Theoretical Study of Nonlinear Dynamics, high-performance computing.

Copyright (c) 2014 by the authors. This is an open access article distributed under the Creative Commons Attribution License (https://creativecommons.org/licenses/by/4.0/), which permits unrestricted use, distribution, and reproduction in any medium, provided the original work is properly cited. 